\title{
Determinants of HIV-1 broadly neutralizing antibody induction
}

\author{
Peter Rusert ${ }^{1,17}$, Roger D Kouyos ${ }^{1,2,17}$, Claus Kadelka ${ }^{1,2}$, Hanna Ebner ${ }^{1}$, Merle Schanz ${ }^{1}$, Michael Huber ${ }^{1}$, \\ Dominique L Braun $^{1,2}$, Nathanael Hozé ${ }^{3}$, Alexandra Scherrer ${ }^{1,2}$, Carsten Magnus ${ }^{1,16}$, Jacqueline Weber ${ }^{1}$, \\ Therese Uhr $^{1}$, Valentina Cippa ${ }^{1}$, Christian W Thorball ${ }^{4,5}$, Herbert Kuster ${ }^{1,2}$, Matthias Cavassini ${ }^{6}$, \\ Enos Bernasconi ${ }^{7}$, Matthias Hoffmann ${ }^{8}$, Alexandra Calmy ${ }^{9}$, Manuel Battegay ${ }^{10}$, Andri Rauch ${ }^{11}$, \\ Sabine Yerly ${ }^{12}$, Vincent Aubert ${ }^{13}$, Thomas Klimkait ${ }^{14}$, Jürg Böni ${ }^{1}$, Jacques Fellay ${ }^{4,5}$, Roland R Regoes ${ }^{4}$, \\ Huldrych F Günthard ${ }^{1,2,18}$, Alexandra Trkola ${ }^{1,18} \&$ the Swiss HIV Cohort Study ${ }^{15}$
}

\begin{abstract}
Broadly neutralizing antibodies (bnAbs) are a focal component of HIV-1 vaccine design, yet basic aspects of their induction remain poorly understood. Here we report on viral, host and disease factors that steer bnAb evolution using the results of a systematic survey in 4,484 HIV-1-infected individuals that identified 239 bnAb inducers. We show that three parameters that reflect the exposure to antigen-viral load, length of untreated infection and viral diversity -independently drive bnAb evolution. Notably, black participants showed significantly $(P=0.0086-0.038)$ higher rates of bnAb induction than white participants. Neutralization fingerprint analysis, which was used to delineate plasma specificity, identified strong virus subtype dependencies, with higher frequencies of CD4-binding-site bnAbs in infection with subtype B viruses $(P=0.02)$ and higher frequencies of V2-glycan-specific bnAbs in infection with non-subtype $B$ viruses $\left(P=1 \times 10^{-5}\right)$. Thus, key host, disease and viral determinants, including subtypespecific envelope features that determine bnAb specificity, remain to be unraveled and harnessed for bnAb-based vaccine design.
\end{abstract}

bnAbs have become blueprints for vaccine design owing to their unequalled activity against divergent HIV-1 strains and proven potency in preventing and suppressing HIV-1 infection after in vivo administration ${ }^{1-8}$. Elicitation of potent bnAb activity is relatively rare in natural HIV-1 infection: only $10-25 \%$ of infected individuals develop breadth, and an estimated $1 \%$ generate highly potent bnAb, or 'elite neutralization', activity ${ }^{9,10}$. Although much is known about the functional properties of bnAbs, the parameters that govern their evolution in natural infection remain unknown, which is a critical limitation for vaccine development. To date, no vaccine approach has induced bn $\mathrm{Ab}$ responses that match those elicited in natural infection ${ }^{1,11}$. Defining what restricts and promotes bnAb evolution in certain individuals will be crucial for devising successful vaccine regimens, as the same restrictions are likely to be encountered during immunization.

Observations that bn $\mathrm{Ab}$ activity arises predominantly in viremic individuals after several years of infection and is linked to lower CD $4^{+}$ cell counts (referred to here as CD4 levels) ${ }^{4,12-14}$ strongly suggest that prolonged exposure to viral antigen is needed for induction of bnAbs.
This may be necessary in part to allow the extensive antibody-affinity maturation that is characteristic of many HIV-1-specific bnAbs ${ }^{15,16}$. Similarly, antigen levels may be relevant, as bnAbs have been found to evolve less frequently in individuals with lower viral loads $1,4,13,17$. Individual case studies delineating pathways of bnAb maturation have highlighted the tight interplay between virus escape and antibody adaptation that precedes the development of a broad neutralization response ${ }^{18-23}$. In line with this, the viral envelope diversity generated by neutralization escape and after superinfection have been suggested to foster bnAb evolution ${ }^{4,18,19,24-26}$. Despite increasing evidence showing a link between bnAb evolution and prospective factors, including immune parameters ${ }^{4,14,27,28}$, the individual influences of these factors remain unclear. The best-established parameters across various studies - length of infection, high viral load, low CD4 level and viral diversity-reflect disease progression and are closely linked, leaving uncertain whether they have independent influences on bnAb evolution. Factors that are indispensable for bnAb evolution or are initial triggers of bnAb induction remain to be determined.

\footnotetext{
${ }^{1}$ Institute of Medical Virology, University of Zurich, Zurich, Switzerland. ${ }^{2}$ Division of Infectious Diseases and Hospital Epidemiology, University Hospital Zurich, Zurich, Switzerland. ${ }^{3}$ Institute of Integrative Biology, Eidgenössische Technische Hochschule (ETH) Zurich, Zurich, Switzerland. ${ }^{4}$ Global Health Institute, School of Life Sciences, École Polytechnique Fédérale de Lausanne, Lausanne, Switzerland. ${ }^{5}$ Swiss Institute of Bioinformatics, Lausanne, Switzerland. ${ }^{2}$ University Hospital Lausanne, University of Lausanne, Lausanne, Switzerland. ${ }^{7}$ Division of Infectious Diseases, Regional Hospital of Lugano, Lugano, Switzerland. ${ }^{8}$ Division of Infectious Diseases, Cantonal Hospital of St. Gallen, St. Gallen, Switzerland. ${ }^{9}$ Division of Infectious Diseases, University Hospital of Geneva, Geneva, Switzerland. ${ }^{10}$ Division of Infectious Diseases, University Hospital of Basel, Basel, Switzerland. ${ }^{11}$ Department of Infectious Diseases, Inselspital, Bern University Hospital, Bern, Switzerland. ${ }^{12}$ Laboratory of Virology, Division of Infectious Diseases, Geneva University Hospital, Geneva, Switzerland. ${ }^{13}$ Division of Immunology and Allergy, University Hospital Lausanne, Lausanne, Switzerland. ${ }^{14}$ Department of Biomedicine-Petersplatz, University of Basel, Basel, Switzerland. ${ }^{15} \mathrm{~A}$ full list of members and affiliations appears at the end of the paper. ${ }^{16}$ Present address: Computational Evolution, Department of Biosystems Science and Engineering (D-BSSE), ETH Zurich, Basel, Switzerland. 17These authors contributed equally to this work. 18These authors jointly directed this work. Correspondence should be addressed to A.T. (trkola.alexandra@virology.uzh.ch) or H.F.G. (huldrych.guenthard@usz.ch).
}

Received 1 March; accepted 25 August; published online 26 September 2016; doi:10.1038/nm.4187 
Similarly, which factors restrict bnAb activity to only a fraction of individuals and influence evolution of bnAb specificity have not been identified. Understanding these determinants will be crucial to promote the triggering of bn $\mathrm{Ab}$ responses by immunization and develop vaccines that are globally effective in individuals from diverse risk groups, ethnicities and HIV-1 subtypes.

\section{RESULTS}

\section{Population-wide survey of bnAb activity}

To perform a systematic investigation of parameters that steer bnAb induction in HIV-1 infection, we analyzed the plasma neutralization activity of 4,484 individuals enrolled in the Swiss HIV Cohort Study (SHCS), founded in 1988 (ref. 29), or the Zurich Primary HIV Infection study (ZPHI), founded in 2002 (ref. 30) (Fig. 1a). These long-term, prospective, observational studies maintain extensive patient and virus data and biobanks of plasma and blood cell samples collected over time, which allowed us to perform a comprehensive analysis of viral, host and disease determinants to explore the parameters that govern the frequency and type of bnAb induction.

From the total 18,489 individuals in the ZPHI and SHCS cohorts, we selected patients with a minimum of 1 year of untreated HIV-1 infection using a range of criteria (Fig. 1a). To probe the influence of long-term antigen exposure, we stratified the cohort according to the duration of infection up to the sampling time point, thereby accounting for times of virus replication during drug-naive and antiretroviraltreatment-interruption periods. Patients were assigned to one of three groups: 1 to $<3$ years of untreated HIV-1 infection (group 1), 3 to $<5$ years of untreated HIV-1 infection (group 3 ) or $\geq 5$ years of untreated HIV-1 infection (group 5), with group numbers indicating the respective minimums of 1,3 and 5 years of infection (Fig. 1a and Supplementary Table 1). Each individual was represented only once, and only plasma samples that showed no nonspecific inhibitory effect (as determined by inhibition of murine leukemia virus infection (MuLV) in a TZM-bl pseudovirus microneutralization assay) were included; this totaled 4,484 plasma samples across the three groups. Specific HIV-1 neutralization activity was probed against a panel of eight HIV-1 pseudovirus strains from diverse subtypes (Supplementary Table 2), which we selected for their differential susceptibility to known bnAb types (Supplementary Tables 3 and 4). This enabled sensitive detection of the corresponding broadly neutralizing plasma responses (Fig. 1b). We recorded the neutralization activity observed for each plasma-virus combination: neutralization $>80 \%$ received a score of 3 , neutralization of $50 \%$ to $<80 \%$ received a score of 2 , neutralization of $20 \%$ to $<50 \%$ received a score of 1 , and neutralization $<20 \%$ received a score of 0 . Plasma samples were then ranked by the sum of scores against all eight viruses to reflect their potency and breadth. The maximum cumulative neutralization score for a given plasma sample was 24 . Scores of $\geq 15$ were categorized as elite neutralization, scores of $10-14$ as broad neutralization, and scores of 5-9 as cross-neutralization (Table 1). Using these thresholds, 699 individuals (15.6\%) showed cross-neutralization activity, 181 individuals (4\%) showed broad neutralization activity, and 58 (1.3\%) showed elite neutralization activity. We next measured neutralization activity of the 105 plasmas with the highest neutralization activity (score $\geq 13$ ) in an extended 40-virus multiclade panel (Fig. 2a and Supplementary Tables 2 and 5). Results from this 40 -virus screen confirmed the validity of the 8 -virus screening panel and the scoring system in identifying individuals with broad (Fig. 2b) and potent (Fig. 2c) neutralizing activity. a
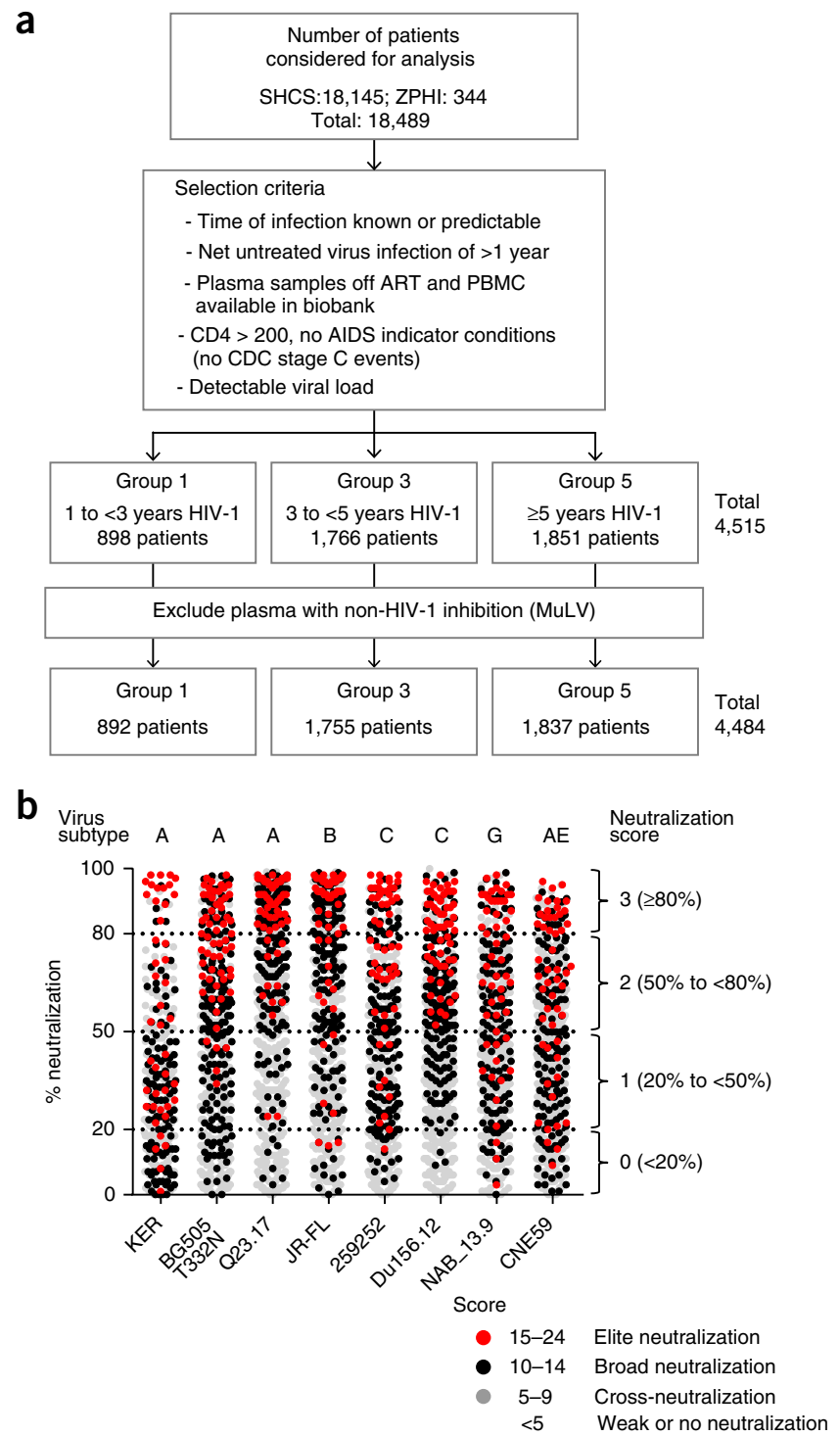

Figure 1 Primary 8-virus neutralization screen identifies 239 bnAb-inducing individuals. (a) Patient selection and stratification (Supplementary Table 1). Plasma samples from 4,484 individuals were included. (b) Results of the primary neutralization screen of 4,484 plasma samples using eight genetically divergent viruses in a TZM-bl-based Env pseudovirus-neutralization assay (Supplementary Tables 2 and 3). Plasma samples were probed at a dilution of $1: 150$. Brackets indicate scoring categories of plasma neutralization activity. Plasmas with elite, broad and cross-neutralization activity are shown as defined in Table 1 . Samples with no or low neutralization activity (cumulative score $<5$ ) are not shown (see Source Data). ART, antiretroviral treatment; PBMC, peripheral blood mononuclear cells; CDC, Centers for Disease Control and Prevention (see Online Methods for details about stage classification).

\section{Defining determinants of bnAb development}

With these large numbers of cross-, broad and elite neutralizers identified and the extensive patient and disease data available in our cohorts, we had a unique opportunity to conduct a systematic analysis of factors that drive bnAb evolution. We focused our analysis on seven parameters, for which we had complete data sets for 4,079 individuals (Fig. 3a): five viral or disease parameters (viral load and CD4 level at the time of sampling, duration of untreated infection, HIV-1 pol subtype and mode of transmission) and two patient-related 
ARTICLES

Table 1 Frequencies of patients with neutralization breadth

\begin{tabular}{lcrc}
\hline & & \multicolumn{2}{c}{ Patients } \\
\cline { 3 - 4 } Neutralization category & Neutralization score & $N$ & $(\%)$ \\
\hline Elite & $15-24$ & 58 & 1.3 \\
Broad & $10-14$ & 181 & 4.0 \\
Cross & $5-9$ & 699 & 15.6 \\
Weak or none & $<5$ & 3,546 & 79.1 \\
\hline
\end{tabular}

aFor each plasma, a cumulative score totaling the neutralization scores against all eight viruses was calculated. Cumulative score thresholds for elite, broad, cross- and weak or no neutralization categories are indicated.

parameters (ethnicity and sex). Each parameter was assessed for its individual association with bnAb induction using univariable and multivariable multinomial logistic regression models controlling for the remaining parameters (Supplementary Table 6). We further verified our findings using linear and Tobit regression models (Supplementary Tables 7-11).

We first explored the impact of viral load and CD4 level on bnAb emergence, two inversely linked parameters that are closely associated with disease progression ${ }^{31}$. Higher viral loads proved to be an independent driver of neutralization breadth (Fig. 3b, Supplementary Fig. 1a,b and Supplementary Table 6), confirming a need for certain levels of antigen exposure. The effect of CD4 levels on broad and elite neutralization was not statistically significant $(P>0.05)$ in our model. Lower CD4 levels were, however, associated with a modest increase in cross-neutralizing activity that was independent of viral load and the other parameters (Fig. 3c, Supplementary Fig. 1c,d and Supplementary Table 6). Notably, this negative effect of CD4 levels was particularly strong in samples of patients with more than $500 \mathrm{CD}^{+}$cells/ $\mu \mathrm{l}$ at the sampling time point (Supplementary Table 8). If there is indeed a direct impact from $\mathrm{CD} 4^{+} \mathrm{T}$ cell loss on bnAb development, this could indicate a decrease in negative regulators of bn $\mathrm{Ab}$ development, potentially caused by enabling survival of auto-reactive $\mathrm{B}$ cells or limiting effects of polyactivation of $\mathrm{B}$ cells ${ }^{12,32,33}$.

As observed previously, the frequency of bnAb inducers strongly increased with infection length, with notably lower activity in group 1 than in groups 3 and 5 (Fig. 3d and Supplementary Fig. 1e,f). However, we found-for the first time, to our knowledge - that the duration of infection was an independent driver of bnAb induction, with exceptionally high association (odds ratio $>3$ for each neutralization category; Fig. 3e). Notably, we found no significant difference between groups 3 and 5 ( $P=0.54$, likelihood ratio test), indicating that the probability for a broad neutralization response does not increase further after several $(\geq 3)$ years of infection in our cohort.

Another parameter closely linked with virus exposure is the genetic diversity of the virus, which is known to increase during the course of the infection ${ }^{34,35}$. We had access to viral-diversity data, based on a method that uses ambiguous nucleotide calls from pol sequences derived during routine genotypic resistance testing (GRT) for antiretroviral treatment (ART) optimization ${ }^{34}$, for 3,306 individuals enrolled in our study. Using pol gene data provides an independent measure of diversity that, unlike env diversity, is not directly influenced by bnAb activity. When adding these GRT-derived data to our multivariate analysis to probe the influence of diversity on bn Ab frequencies, we also controlled for the time points of testing, as these varied among

a
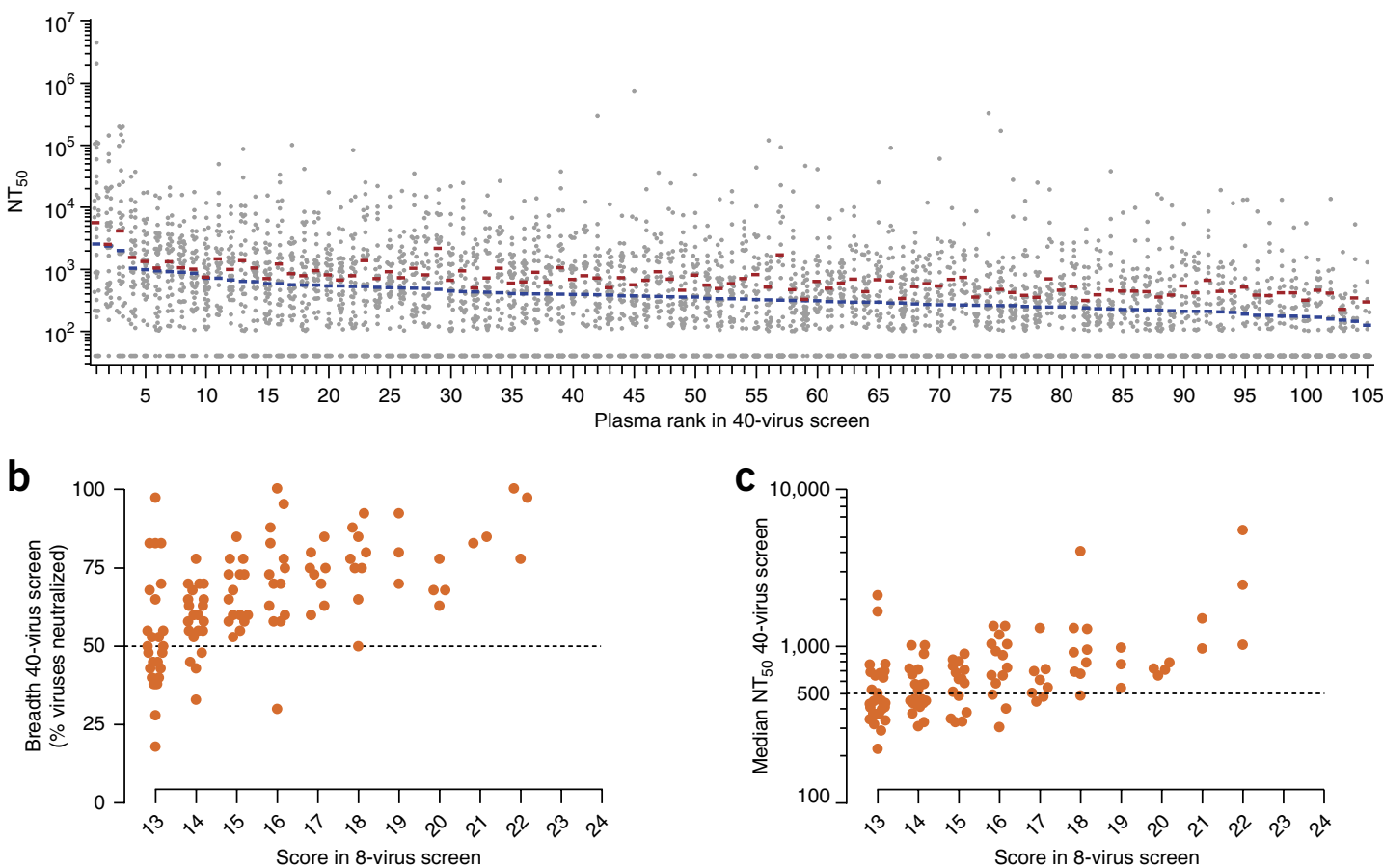

Figure 2 Primary 8-virus screen detects broad and potent neutralization. (a) Verification of neutralization breadth and potency of the 105 top scoring plasmas (cumulative score $\geq 13$; Supplementary Tables 2 and $\mathbf{5}$ ). Plasma samples are ranked according to the geometric mean $50 \%$ neutralization titer $\left(\mathrm{NT}_{50}\right.$ ) determined across neutralized and non-neutralized strains (blue bars). Geometric mean $\mathrm{NT}_{50}$ values determined solely across neutralized strains are also shown (red bars). (b,c) Comparison of the neutralization score in the primary screen with breadth (b) and potency (c) determined in the 40-virus secondary screen (Supplementary Table 5). Breadth (b) is defined by the percentage of strains neutralized at titers $>1: 100$ in the 40-virus screen; potency (c) is defined by the median $\mathrm{NT}_{50}$ values across all tested strains (Supplementary Table 5 ). Dashed line indicates $>50 \%$ neutralization breadth (b) or high potency $\left(\mathrm{NT}_{50}>1: 500\right)$ (c). 
a

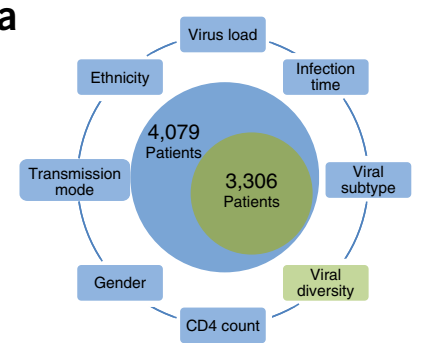

b

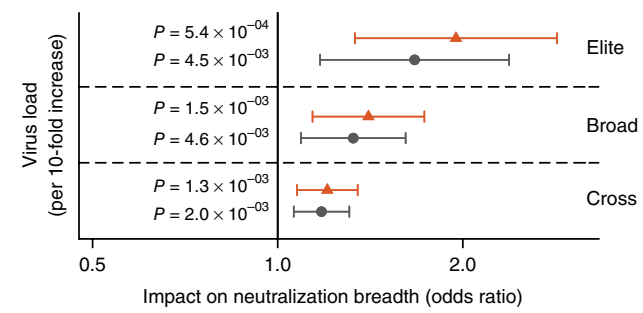

C

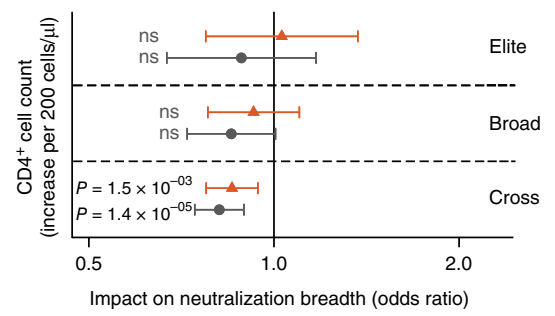

d

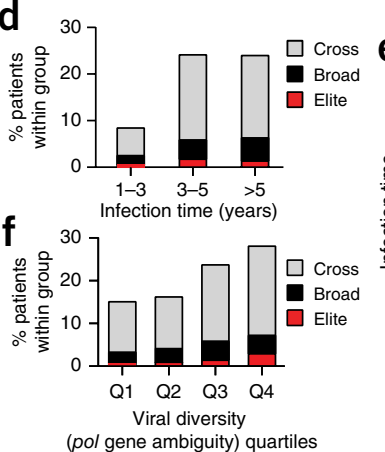

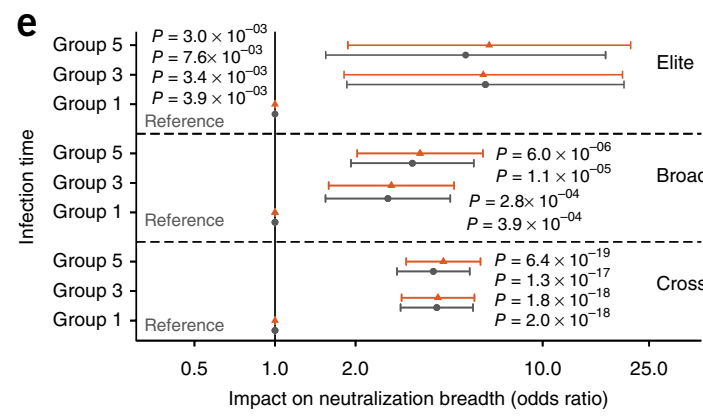

g

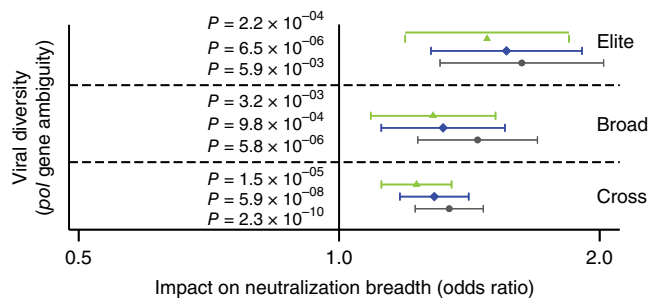

Figure 3 Influence of viral and disease parameters on the development of neutralization breadth. (a) Overview of the parameters tested for influence on neutralization breadth and the numbers of individuals for which full data sets were available. (b-f) Influence of virus load (b), effects of CD4+ cell count increase per 200 cells (c) and duration of untreated infection (e) on the development of neutralization breadth, and distribution of plasmas showing cross-, broad and elite neutralization activity across patient groups with different infection times (d) or degrees of viral diversity (quartile 1 , lowest; quartile 4, highest) as assessed by sequence diversity in pol (f). For b, $\mathbf{c}$ and e, odds ratios and $95 \%$ confidence intervals for the indicated parameters in the univariable (gray) and multivariable (orange) multinomial logistic regression analyses and significant $P$ values are shown. RNA copies per $\mathrm{ml}$ blood (b) and $\mathrm{CD}^{+}{ }^{+}$cells per $\mu$ l blood (c) were used as continuous variables. (g) Univariable analysis with unadjusted (gray) and time-adjusted

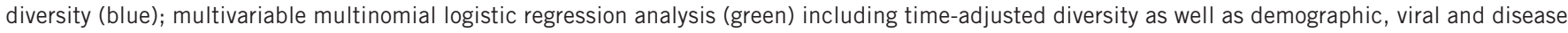
parameters (Supplementary Table 6 and Source Data). ns, no significance.

individuals. The odds ratios we obtained confirmed that increasing diversity is a third independent factor associated with antigen exposure that influences the development of breadth (Fig. 3f,g, Supplementary Fig. 1g,h and Supplementary Table 6). A significant and strong effect of diversity persisted even after adjusting for the time of both the diversity estimate and the neutralization screen (Fig. 3g), indicating that the impact of diversity was independent of the duration of infection and the time point of GRT. This influence of diversity supports the observation of cases with bnAb evolution after superinfection ${ }^{19,24}$ and underscores the potential of genetically diverse prime-boost vaccine regimens.

The SHCS and ZPHI cohorts reflect the HIV-1 epidemic in Switzerland, which is dominated by subtype B infection in white individuals (Fig. 4a-c). Non-subtype B infections represented $24 \%$ of the 4,484 study participants. Subtypes A, circulating recombinant form (CRF) 02_AG, subtype C and CRF01_AE, as determined from pol sequence data, were the largest groups outside subtype $B$ and were more frequent among nonwhite participants (Fig. $\mathbf{4 a - c}$ ). We thus probed the influence of virus subtype and ethnicity on the frequency of bnAb induction. We observed no statistical difference in the frequency of broad neutralization development between subtype B with non-subtype B infections (Fig. 4d,e) or between individual non-B subtypes (Supplementary Table 9). Notably, however, black ethnicity consistently showed a strong and independent effect on bnAb induction, as compared to white ethnicity (Fig. 4f,g and Supplementary Table 9). Differences in the studied ethnic groups may be due to a range of factors. For example, infection characteristics in our cohort differed among ethnicities: nonwhite participants acquired HIV-1 more frequently outside of Switzerland, their infection was detected later and they showed higher frequencies of females and heterosexual transmissions ${ }^{36,37}$ (Supplementary Table 12). Controlling for confounding parameters, our analysis confirmed, however, that black ethnicity is associated with higher bnAb frequencies independently of influences from all other probed variables, including the duration of infection (Supplementary Table 11). This association with black ethnicity merits further exploration, but definition of factors that generally influence bnAb induction and which are more frequently represented among black individuals and how they are represented in other ethnicities will require additional studies specifically tailored to address differences among ethnicities. In principle, many factors, including the geographical and socioeconomic setting of HIV-1 acquisition and host genomics, may affect infection and thereby influence immune responses. Consequently, a range of additional factors (for example, coinfection with other sexually transmitted diseases at the time of HIV-1 acquisition) that are not systematically recorded for the majority of infected individuals need to be considered, as they may have roles in stimulating antibody responses. The black individuals in our cohort have highly diverse backgrounds, including Sub-Saharan Africa, Northern Africa, North America or the Caribbean, and most of them acquired HIV-1 infection abroad ${ }^{37}$ (Supplementary Table 13). A higher propensity to mount neutralization breadth was observed across black participants (Supplementary Fig. 2) and did not depend on whether infection dates were confirmed or estimated (Supplementary Table 10). Although this may suggest that geographical or socioeconomic factors at time of infection (for example, access to health care) are not the main drivers of the observed differences between black and white individuals in this cohort, our study was not tailored to address these parameters, thus we cannot rule out a contribution from such influences. Of particular note, a recent vaccine trial observed higher neutralization titers 


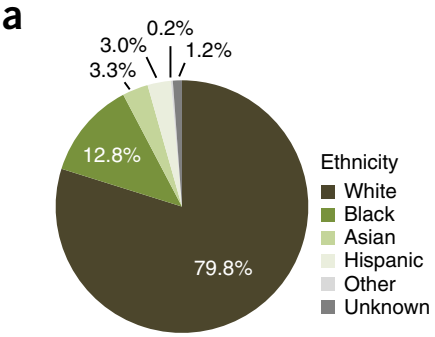

b

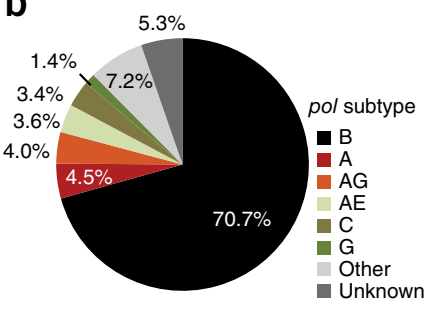

Viral load (per 10-fold increase)
Viral diversity (based on pol)
Infection time (3-5 years versus $<3$ years)
Infection time ( $>5$ years versus $<3$ years)
CD4 (decrease per 200)
Black ethnicity
pol subtype (non-B versus B)
Transmission mode (HET versus MSM)
Gender (female versus male)

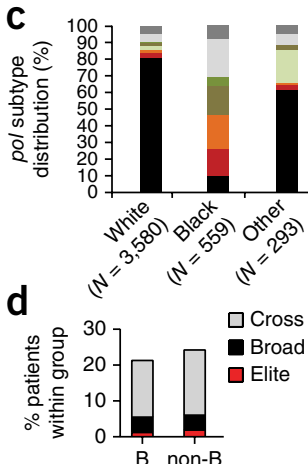

B non-B

Infecting subtype (pol)

f
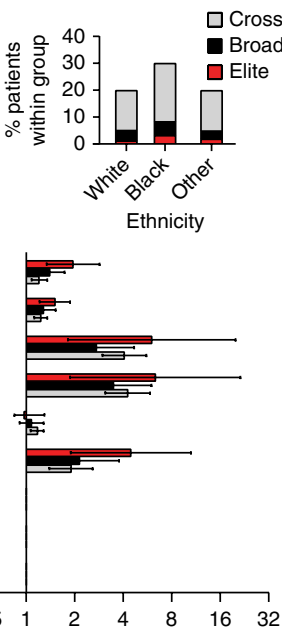

e
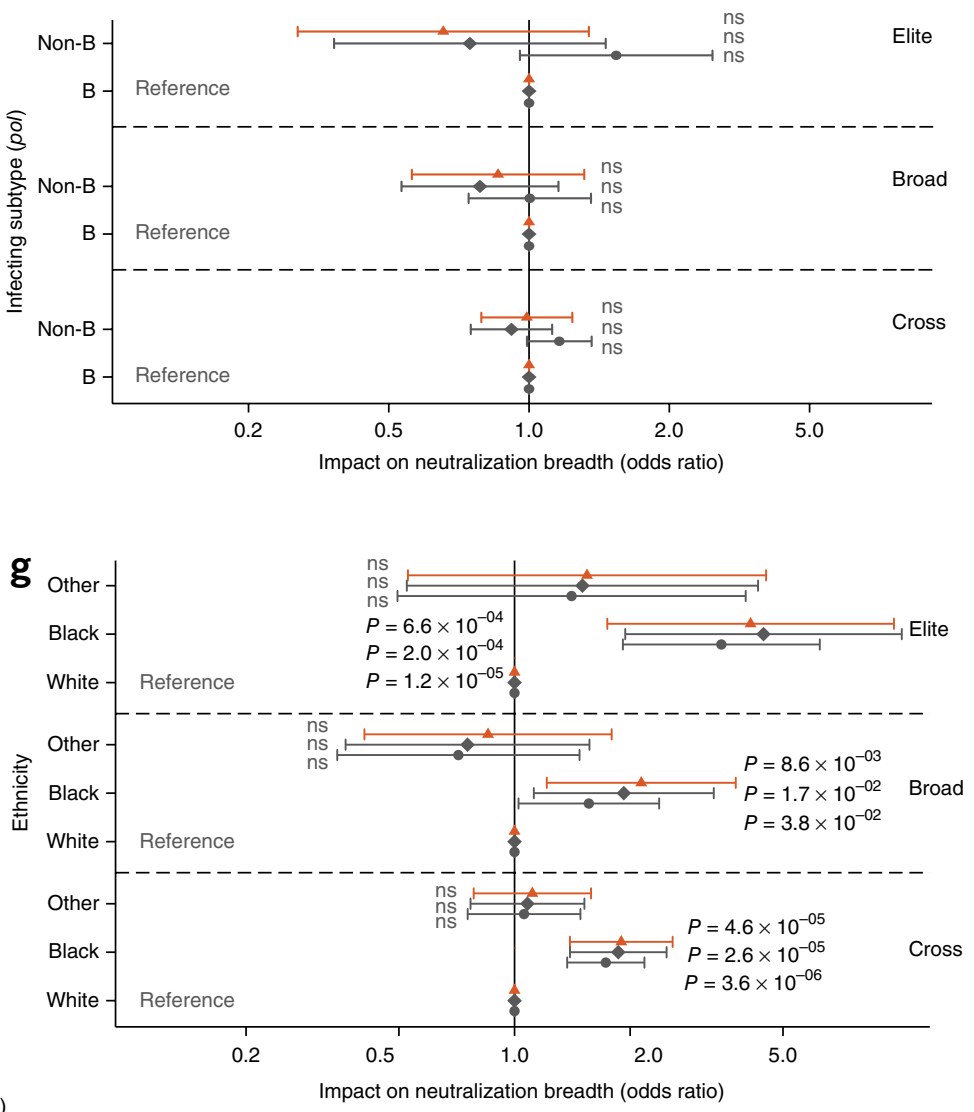

Figure 4 Ethnicity influences the development of neutralization breadth. (a,b) Distribution of ethnicities (a) and HIV-1 pol subtypes (b) in our cohort. (c) Distribution of HIV-1 po/ subtypes across ethnicities. (d,e) Distribution of cross-, broad and elite neutralizing plasmas across subtypes (d) and influence of subtype on neutralization breadth development $(\mathbf{e}) .(\mathbf{f}, \mathbf{g})$ Distribution of cross-, broad and elite neutralizing plasmas across ethnicities $(\mathbf{f})$ and influence of ethnicity on neutralization breadth development (g). Impact on neutralization breadth was determined by multinomial logistic regression and odds ratios with breadth of neutralization response as the outcome variable. Shown are odds ratios and $95 \%$ confidence intervals for univariable model (gray circles), multivariable model including ethnicity (e) and subtype (g) (gray diamonds) and multivariable model including all parameters (orange).

(h) Summary of odds ratios and confidence intervals for all probed parameters (Supplementary Table 6 and Source Data). ns, no significance.

among African Americans than whites in response to a gp120 vaccine ${ }^{38}$, indicating the existence of ethnicity-dependent variations among drivers of antibody induction.

Notably, in our cohort, ethnicity was, after duration of infection, the strongest driver of neutralization breadth (Fig. 4h). An analysis of the remaining parameters considered in our study (sex and mode of transmission) revealed no additional, consistent influences on bn $\mathrm{Ab}$ evolution (Fig. 4h and Supplementary Table 6).

\section{Virus subtype influences prevalence of bnAb types}

We next asked whether there are specific factors that guide the epitope specificity of bnAbs. Delineation of bnAb specificity in polyclonal plasma is complex, and both mathematical prediction, based on bnAb neutralization fingerprint analysis, and mapping with mutant virus devoid of known bnAb epitopes are often only partially successful in defining specificities ${ }^{14,24,39,40}$. We relied here on a comparison of prediction methods to deconvolute the dominant neutralization specificity of the 105 top neutralizing plasmas analyzed for neutralization activity against the 40 -virus multiclade panel (Fig. 2 and Supplementary Table 5). To delineate specificities, we used neutralization fingerprints of known bnAbs covering the major neutralization-sensitive sites on the viral envelope against the same 40-virus panel (Supplementary Table 4). Focusing solely on the bnAbs that neutralize $>50 \%$ of the probed viruses, we established a strategy, termed maximal-Spearman-based prediction (MSBP), to define specificities in plasmas that match a known bnAb type. The prediction yielded a dominant bnAb type for 82 of the 105 plasmas (Fig. 5a,b, Supplementary Fig. 3 and Supplementary Data Set 1). We verified the MSBP-based specificity prediction in a sensitivity analysis using three alternative methods, including a delineation algorithm developed recently by Georgiev et al. ${ }^{40}$ (Supplementary Figs. 4 and 5 and Supplementary Data Sets 2 and 3). All four prediction methods yielded highly similar results in predicting CD4 binding site (CD4bs) and V2-glycan-like responses but differed in dissection of V3-glycan and membrane-proximal external region (MPER) specificities, suggesting that these predictions are less accurate (Supplementary Figs. $4 a-c$ and $\mathbf{5 a}-\mathbf{c}$ ). To further verify the validity of the computational epitope delineation, we confirmed that plasma samples predicted to contain V2-glycan specificities lost neutralization activity in response to the same V2-mutant viruses as known V2-glycan bnAbs (Supplementary Fig. 6 and Supplementary Data Set 4).

We next investigated whether the infecting subtype has an influence on the type of bnAb response elicited. When we compared the bnAb specificities of plasmas originating from subtype B and non-B infections, 
a

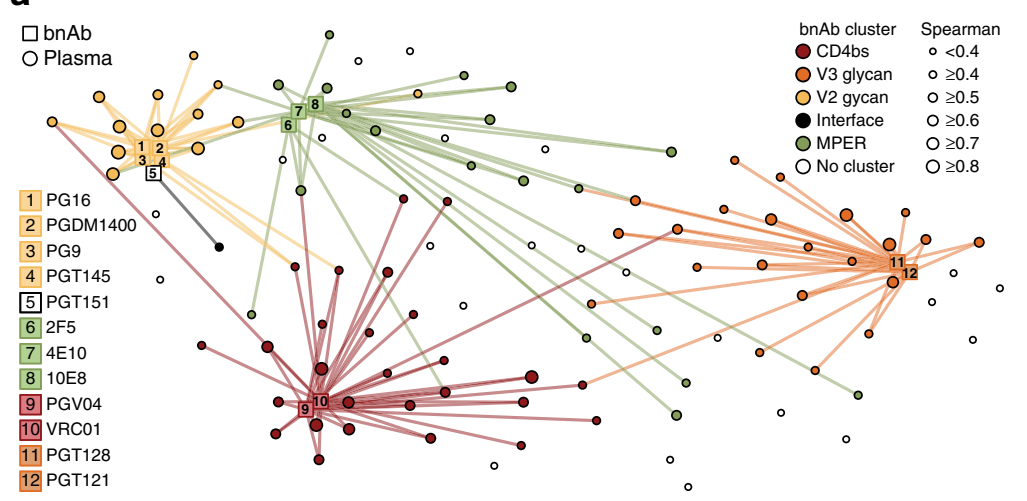

C

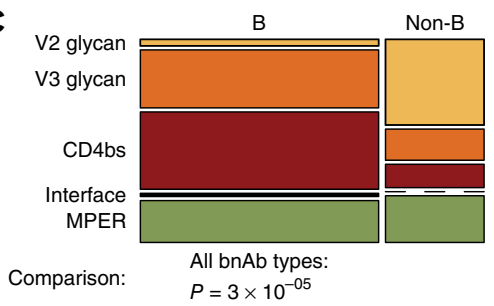

d

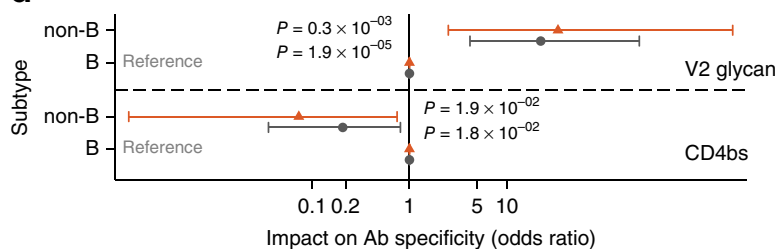

b

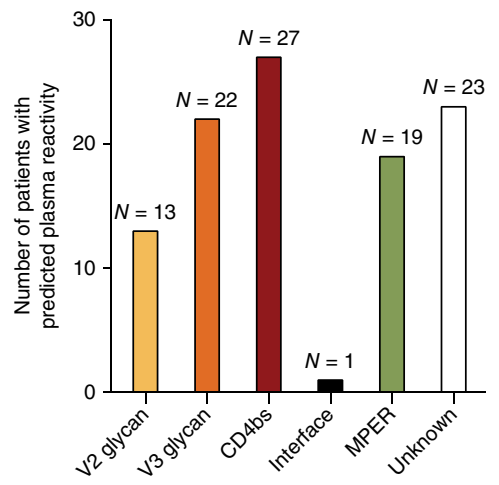

B
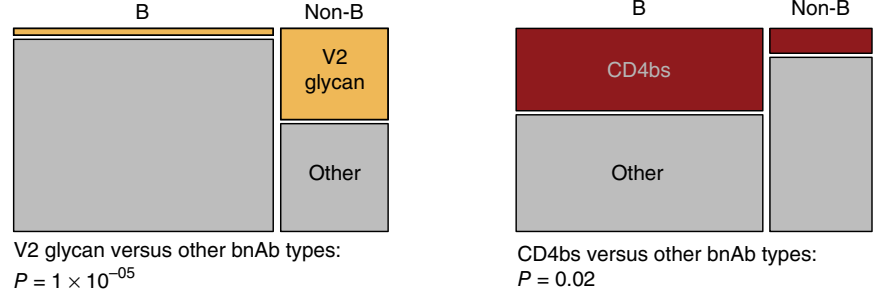

CD4bs versus other bnAb types: $P=0.02$

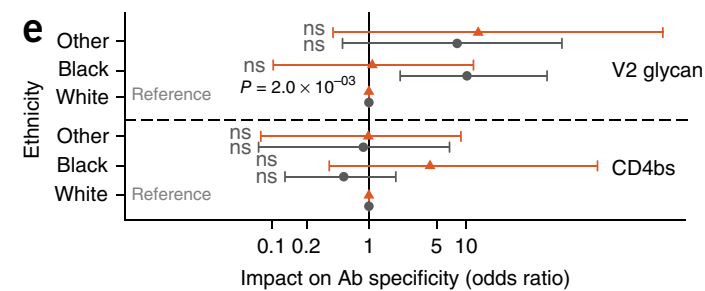

Figure 5 Influence of HIV-1 subtype on the type of bnAb response. (a-e) Analysis of bnAb specificity according to MSBP. (a) Predicted dominant bnAb specificity in the 105 top neutralizing plasma samples (Fig. 2, Supplementary Figs. 3-6 and Supplementary Table 5). Known bnAbs (squares) and plasma samples (circles) are plotted using a weighted version of $t$-distributed stochastic neighbor embedding with the Spearman correlation of neutralization activity in the 40-virus screen as the pairwise distance measure. Lines connect known bnAbs and plasma samples with Spearman correlation $>0.4$. Plasma sample data are colored according to dominant bnAb specificity. No cluster, plasma samples for which prediction of a dominant specificity was not possible. (b) Summary of predicted bnAb specificities. (c) Association of infecting HIV-1 pol subtype and bnAb specificity probed by Fisher's exact test. Rectangle sizes correspond to the proportion of included plasma samples (Supplementary Figs. $\mathbf{4}$ and $\mathbf{5}$ ). (d,e) The influence of subtype (d) and ethnicity (e) on the development of V2-glycan and CD4bs bnAb specificities, determined using exact logistic regression with the type of bnAb response as the outcome variable (Supplementary Table 14). Shown are odds ratios and $95 \%$ confidence intervals for the indicated parameters in the univariable (gray) and multivariable (orange) setting controlling for ethnicity in (d) and subtype in (e). ns, no significance.

we found that the distribution of bnAb specificities differed significantly across all prediction methods (Fig. 5c and Supplementary Figs. 4d and 5d). Plasmas with V2-glycan-specific antibodies were dominated by non-subtype $\mathrm{B}$ infection $\left(P=1 \times 10^{-5}\right.$ with MSBP; $P=8 \times 10^{-6}$ to $1 \times 10^{-3}$ with other methods). Conversely, CD4bsspecific bnAbs were more prevalent in subtype B infection $(P=0.02$ with MSBP; $P=8 \times 10^{-4}$ to 0.04 with other methods; Fig. $5 \mathrm{c}$ and Supplementary Figs. 4d and 5d). In contrast, there was no consistent significant difference between subtype $B$ and non-B infections in inducing V3-glycan and MPER responses. Owing to the lower resolution in defining these two specificities (Supplementary Figs. 4b,c and $\mathbf{5 b}, \mathbf{c})$, however, this result must be interpreted with caution. The underrepresentation of V2-glycan responses in subtype B infection was notable, especially when considering that the 8-virus panel contained several strains highly sensitive to known V2-glycan bnAbs (Supplementary Table 4). Suggesting an unfavorable presentation of the V2-glycan epitope in subtype B viruses, this finding may also explain why potent V2-glycan bnAbs have thus far been identified only in non-B infection (Supplementary Table 3). Notably, the infecting subtype steered both CD4bs and V2-glycan responses independently of ethnicity (Fig. 5d,e and Supplementary Table 14). By contrast, ethnicity had no significant effect on the type of antibody response in the multivariable model. Although influences of viral subtype on the frequency of bnAb elicitation may be subtle and our study may have lacked the power to identify these, the viral subtype proved a driver of the type of antibody response that is evoked.

\section{DISCUSSION}

Our cohorts contain a large number of individuals with detailed demographic and disease parameters. Through this populationwide survey we were able to put the increasing number of parameters considered to be drivers of neutralization breadth into perspective, explore their interdependencies and identify novel parameters. We found that a range of factors, including some that have been suggested before, independently promote the evolution of broad neutralization responses, and this result offers exciting opportunities for vaccine design. Antigen load, duration of viral exposure and viral diversity link bnAb evolution to extended antigen experience. They can, therefore, be exploited individually in promoting bn Ab induction in vaccine regimens. Years of antigen contact that favor 
bnAb evolution in natural infection cannot be replicated by vaccination. Our findings lend support to the idea that prolonged antigen exposure, although an influential driver of bnAb evolution, may be dispensable if other factors that promote bnAb elicitation are provided. This is also supported by individual case reports, including cases of mother-to-child transmission in which bnAb activity develops early after infection ${ }^{41}$, suggesting that effective elicitation of bnAbs by vaccination should, in principle, be possible.

The pronounced influence of ethnicity on frequency of bnAb induction is intriguing and raises the possibility that specific factors drive bnAb evolution. Definition of these variables and their prevalence across ethnicities will be key and will help to determine whether any might open new roads for stimulation of bnAb responses. It is important to note that our study does not have the means to dissect host genetics or socioeconomic or geographical parameters that may drive the influence of ethnicity on bnAb evolution. Determining the precise links between ethnicity and likelihood of developing bnAbs will require extensive study of bnAb inducers from diverse ethnicities and may specifically require large-scale surveys across black individuals with different genetic and geographical backgrounds.

Multispecificity of neutralizing antibody responses is considered crucial for sustained vaccine efficacy across HIV-1 subtypes and requires detailed knowledge about how to elicit individual bn $\mathrm{Ab}$ responses. Here we show for V2-glycan and CD4bs bnAbs that the type of bnAb induced strongly depends on the subtype of the infecting virus. This result suggests that the viral envelope proteins harbor subtype specific conformational traits that favor the evolution of one specificity over another. To date, the structural information on native HIV-1 envelope trimer conformation is limited to a few selected strains ${ }^{42}$. Broader comparisons of envelope structures across viral subtypes are therefore necessary to identify the determinants of the preferential induction of V2-glycan, CD4bs and possibly other bnAb specificities to exploit them for immunogen design. The identification of these determinants of bnAb specificity, together with the definition of potential disease, socioeconomic, geographical and host genomic factors involved in bnAb evolution as well as parameters that replicate important features of HIV-1 antigen exposure in immunization will open new avenues for rational HIV-1 vaccine design.

\section{METHODS}

Methods and any associated references are available in the online version of the paper.

Note: Any Supplementary Information and Source Data files are available in the online version of the paper.

\section{ACKNOWLEDGMENTS}

We thank the patients participating in the ZPHI and the SHCS and their physicians and study nurses for patient care; B. Remy, F. Schoeni-Affolter and Y. Vallet from the SHCS Data Center in Lausanne for data management; M. Robbiani, D. Perraudin and M. Minichiello for administrative assistance. We thank D. Burton (the Scripps Research Institute), J. Mascola (US National Institutes of Health (NIH) Vaccine Research Center), M. Nussenzweig (Rockefeller University), M. Connors (NIH), D. Katinger (Polymun), P. Moore and L. Morris (Center for Communicable Diseases, South Africa) for providing antibodies and/or antibody expression plasmids for this study, either directly or via the NIH AIDS Research and Reference Reagent Program. Financial support for this study was provided by the Swiss National Science Foundation (grant 310030_152663 to A.T.), the Clinical Priority Research Priority Program of the University of Zurich (viral infectious diseases: Zurich Primary HIV Infection Study to H.F.G. and A.T.), the Yvonne-Jacob Foundation (to H.F.G.) the Swiss Vaccine Research Institute (to A.T., H.F.G., R.D.K., R.R.R. and J.F.) and a SystemsX.ch grant (AntibodyX to A.T. and R.R.R.). R.D.K. was supported by the Swiss National Science Foundation (PZ00P3-142411 and BSSGI0_155851). This study was cofunded within the framework of the Swiss
HIV Cohort Study, supported by the Swiss National Science Foundation (grant 33CS30_148522 to H.F.G.), the small nested SHCS project 744 (to A.T.) and the SHCS research foundation. The funders had no role in study design, data collection and analysis, decision to publish or preparation of the manuscript.

\section{AUTHOR CONTRIBUTIONS}

P.R., R.D.K., H.F.G. and A.T. conceived and designed the study and analyzed data. P.R., H.E., M.S., M. Huber, C.W.T. and J.F. designed and performed experiments and analyzed data. J.W., T.U., V.C., H.K., S.Y., V.A., T.K. and J.B. conducted experiments and analyzed data. C.K., A.S., C.M., N.H. and R.R.R. conducted computational analyses and contributed analysis tools and data analysis. D.L.B., M.C., E.B., M. Hoffmann, A.C., M.B., A.R., H.F.G. and the members of the Swiss HIV Cohort Study conceived and managed the SHCS and ZPHI cohorts collected and contributed patient samples and clinical data. P.R., R.D.K., M.S., C.K., M. Huber, H.F.G. and A.T. wrote the manuscript, which all coauthors commented on.

\section{COMPETING FINANCIAL INTERESTS}

The authors declare no competing financial interests.

Reprints and permissions information is available online at http://www.nature.com/ reprints/index.html.

1. Burton, D.R. \& Mascola, J.R. Antibody responses to envelope glycoproteins in HIV-1 infection. Nat. Immunol. 16, 571-576 (2015).

2. Klein, F. et al. Antibodies in HIV-1 vaccine development and therapy. Science $\mathbf{3 4 1}$, 1199-1204 (2013).

3. Mascola, J.R. \& Haynes, B.F. HIV-1 neutralizing antibodies: understanding nature's pathways. Immunol. Rev. 254, 225-244 (2013).

4. Moore, P.L., Williamson, C. \& Morris, L. Virological features associated with the development of broadly neutralizing antibodies to HIV-1. Trends Microbiol. 23, 204-211 (2015).

5. Caskey, M. et al. Viremia suppressed in HIV-1-infected humans by broadly neutralizing antibody 3BNC117. Nature 522, 487-491 (2015).

6. Lynch, R.M. et al. Virologic effects of broadly neutralizing antibody VRCO1 administration during chronic HIV-1 infection. Sci. Transl. Med. 7, 319ra206 (2015).

7. Trkola, A. et al. Delay of HIV-1 rebound after cessation of antiretroviral therapy through passive transfer of human neutralizing antibodies. Nat. Med. 11, 615-622 (2005).

8. Scheid, J.F. et al. HIV-1 antibody 3BNC117 suppresses viral rebound in humans during treatment interruption. Nature 535, 556-560 (2016).

9. Euler, Z. et al. Longitudinal analysis of early HIV-1-specific neutralizing activity in an elite neutralizer and in five patients who developed cross-reactive neutralizing activity. J. Virol. 86, 2045-2055 (2012).

10. Simek, M.D. et al. Human immunodeficiency virus type 1 elite neutralizers: individuals with broad and potent neutralizing activity identified by using a highthroughput neutralization assay together with an analytical selection algorithm. J. Virol. 83, 7337-7348 (2009).

11. Burton, D.R. et al. A blueprint for HIV vaccine discovery. Cell Host Microbe 12 396-407 (2012).

12. Gray, E.S. et al. The neutralization breadth of HIV-1 develops incrementally over 4 years and is associated with $\mathrm{CD} 4^{+} \mathrm{T}$ cell decline and high viral load during acute infection. J. Virol. 85, 4828-4840 (2011).

13. Sather, D.N. et al. Factors associated with the development of cross-reactive neutralizing antibodies during human immunodeficiency virus type 1 infection. J. Virol. 83, 757-769 (2009).

14. Landais, E. et al. Broadly neutralizing antibody responses in a large longitudinal sub-Saharan HIV primary infection cohort. PLoS Pathog. 12, e1005369 (2016).

15. Klein, F. et al. Somatic mutations of the immunoglobulin framework are generally required for broad and potent HIV-1 neutralization. Cell 153, 126-138 (2013).

16. Kwong, P.D. \& Mascola, J.R. Human antibodies that neutralize HIV-1: identification, structures and B cell ontogenies. Immunity 37, 412-425 (2012).

17. Doria-Rose, N.A. et al. Breadth of human-immunodeficiency-virus-specific neutralizing activity in sera: clustering analysis and association with clinical variables. J. Virol. 84, 1631-1636 (2010).

18. Moore, P.L. et al. Evolution of an HIV glycan-dependent broadly neutralizing antibody epitope through immune escape. Nat. Med. 18, 1688-1692 (2012).

19. Bhiman, J.N. et al. Viral variants that initiate and drive maturation of V1V2-directed HIV-1 broadly neutralizing antibodies. Nat. Med. 21, 1332-1336 (2015).

20. Doria-Rose, N.A. et al. Developmental pathway for potent V1V2-directed HIVneutralizing antibodies. Nature 509, 55-62 (2014).

21. Liao, H.X. et al. Coevolution of a broadly neutralizing HIV-1 antibody and founder virus. Nature 496, 469-476 (2013).

22. Wu, X. et al. Focused evolution of HIV-1 neutralizing antibodies revealed by structures and deep sequencing. Science 333, 1593-1602 (2011).

23. Wibmer, C.K. et al. Viral escape from HIV-1 neutralizing antibodies drives increased plasma neutralization breadth through sequential recognition of multiple epitopes and immunotypes. PLoS Pathog. 9, e1003738 (2013).

24. Cortez, V. et al. The broad neutralizing antibody responses after HIV-1 superinfection are not dominated by antibodies directed to epitopes common in single infection. PLoS Pathog. 11, e1004973 (2015). 
25. Luo, S. \& Perelson, A.S. Competitive exclusion by autologous antibodies can prevent broad HIV-1 antibodies from arising. Proc. Natl. Acad. Sci. USA 112, 1165411659 (2015).

26. Piantadosi, A. et al. Breadth of neutralizing antibody response to human immunodeficiency virus type 1 is affected by factors early in infection but does not influence disease progression. J. Virol. 83, 10269-10274 (2009).

27. Derdeyn, C.A., Moore, P.L. \& Morris, L. Development of broadly neutralizing antibodies from autologous neutralizing antibody responses in HIV infection. Curr. Opin. HIV AIDS 9, 210-216 (2014).

28. Locci, M. et al. Human circulating PD- $1^{+} \mathrm{CXCR} 3^{-} \mathrm{CXCR} 5^{+}$memory $\mathrm{T}_{\mathrm{FH}}$ cells are highly functional and correlate with broadly neutralizing HIV antibody responses. Immunity 39, 758-769 (2013)

29. Schoeni-Affolter, F. et al. Cohort profile: the Swiss HIV Cohort study. Int. J. Epidemiol. 39, 1179-1189 (2010).

30. Rieder, P. et al. Characterization of human immunodeficiency virus type 1 (HIV-1) diversity and tropism in 145 patients with primary HIV-1 infection. Clin. Infect. Dis. 53, 1271-1279 (2011).

31. Mellors, J.W. et al. Plasma viral load and CD4+ lymphocytes as prognostic markers of HIV-1 infection. Ann. Intern. Med. 126, 946-954 (1997).

32. Recher, M. et al. Deliberate removal of $T$ cell help improves virus-neutralizing antibody production. Nat. Immunol. 5, 934-942 (2004).

33. Kelsoe, G., Verkoczy, L. \& Haynes, B.F. immune system regulation in the induction of broadly neutralizing HIV-1 antibodies. Vaccines (Basel) 2, 1-14 (2014).
34. Kouyos, R.D. et al. Ambiguous nucleotide calls from population-based sequencing of HIV-1 are a marker for viral diversity and the age of infection. Clin. Infect. Dis. 52, 532-539 (2011).

35. Shankarappa, R. et al. Consistent viral evolutionary changes associated with the progression of human immunodeficiency virus type 1 infection. J. Virol. 73 10489-10502 (1999).

36. Kouyos, R.D. et al. Molecular epidemiology reveals long-term changes in HIV type 1 subtype B transmission in Switzerland. J. Infect. Dis. 201, 1488-1497 (2010).

37. von Wyl, $\mathrm{V}$. et al. The role of migration and domestic transmission in the spread of HIV-1 non-B subtypes in Switzerland. J. Infect. Dis. 204, 1095-1103 (2011).

38. Montefiori, D.C. et al. Demographic factors that influence the neutralizing antibody response in recipients of recombinant HIV-1 gp120 vaccines. J. Infect. Dis. 190, 1962-1969 (2004).

39. Chuang, G.Y. et al. Residue-level prediction of HIV-1 antibody epitopes based on neutralization of diverse viral strains. J. Virol. 87, 10047-10058 (2013).

40. Georgiev, I.S. et al. Delineating antibody recognition in polyclonal sera from patterns of HIV-1 isolate neutralization. Science 340, 751-756 (2013).

41. Goo, L., Chohan, V., Nduati, R. \& Overbaugh, J. Early development of broadly neutralizing antibodies in HIV-1-infected infants. Nat. Med. 20, 655-658 (2014).

42. Ward, A.B. \& Wilson, I.A. Insights into the trimeric HIV-1 envelope glycoprotein structure. Trends Biochem. Sci. 40, 101-107 (2015).

\title{
The Swiss HIV Cohort Study
}

\author{
Heiner C Bucher ${ }^{1}$, Angela Ciuffi ${ }^{2}$, Günther Dollenmaier ${ }^{3}$, Matthias Egger ${ }^{4}$, Luigia Elzi ${ }^{5}$, Jan Fehr ${ }^{6}$, \\ Hansjakob Furrer ${ }^{7}$, Christoph A Fux ${ }^{8}$, David Haerry ${ }^{9}$, Barbara Hasse ${ }^{6}$, Hans H Hirsch $^{5,10}$, Irene Hösli ${ }^{11}$, \\ Christian Kahlert ${ }^{12,13}$, Laurent Kaiser ${ }^{14,15}$, Olivia Keiser ${ }^{4}$, Helen Kovari ${ }^{6}$, Bruno Ledergerber ${ }^{6}$, Gladys Martinetti ${ }^{16}$, \\ Begoña Martinez de Tejada ${ }^{17}$, Catia Marzolini ${ }^{5}$, Karin J Metzner ${ }^{6,18}$, Nicolas Müller ${ }^{6}$, Dunja Nicca ${ }^{12,19}$, \\ Giuseppe Pantaleo $^{20}$, Paolo Paioni ${ }^{21}$, Christoph Rudin ${ }^{22}$, Patrick Schmid ${ }^{12}$, Roberto Speck ${ }^{6}$, Marcel Stöckle ${ }^{5}$, \\ Philip Tarr ${ }^{23}$, Pietro Vernazza ${ }^{12}$, Gilles Wandeler ${ }^{7} \&$ Rainer Weber ${ }^{6}$
}

\footnotetext{
${ }^{1}$ Basel Institute for Clinical Epidemiology and Biostatistics, University Hospital Basel, Basel, Switzerland. ${ }^{2}$ Institute of Medical Microbiology, University Hospital Lausanne, Lausanne, Switzerland. ${ }^{3}$ Centre for Laboratory Medicine, Canton St. Gallen, St. Gallen, Switzerland. ${ }^{4}$ Institute of Social and Preventive Medicine, University of Bern, Bern, Switzerland. ${ }^{5}$ Division of Infectious Diseases and Hospital Epidemiology, University Hospital of Basel, Basel, Switzerland. ${ }^{6}$ Division of Infectious Diseases and Hospital Epidemiology, University Hospital Zurich, Zurich, Switzerland. ${ }^{7}$ Department of Infectious Diseases, Inselspital, Bern University Hospital, Bern, Switzerland. ${ }^{8}$ Clinic for Infectious Diseases and Hospital Hygiene, Cantonal Hospital Aarau, Aarau, Switzerland. ${ }^{9}$ Positive Council Switzerland, Zurich, Switzerland. ${ }^{10}$ Division of Infection Diagnostics, Department of Biomedicine-Petersplatz, University of Basel, Basel, Switzerland. ${ }^{11}$ Clinic for Obstetrics, University Hospital Basel, Basel, Switzerland. 12Division of Infectious Diseases and Hospital Epidemiology, Cantonal Hospital St. Gallen, St. Gallen, Switzerland ${ }^{13}$ Children's Hospital of Eastern Switzerland, St. Gallen, Switzerland. 14Division of Infectious Diseases, University Hospital Geneva, Geneva, Switzerland. ${ }^{15}$ Laboratory of Virology, University Hospital Geneva, Geneva, Switzerland. ${ }^{16}$ Cantonal Institute of Microbiology, Bellinzona, Switzerland. ${ }^{17}$ Department of Obstetrics and Gynecology, University Hospital Geneva, Geneva, Switzerland. ${ }^{18}$ Institute of Medical Virology, University of Zurich, Zurich, Switzerland. 19 Institute of Nursing Science, University of Basel, Basel, Switzerland. ${ }^{20}$ Division of Immunology and Allergy, University Hospital Lausanne, Lausanne, Switzerland. 21 University Children's Hospital, University of Zurich, Zurich, Switzerland. ${ }^{22}$ University Children's Hospital, University of Basel, Basel, Switzerland. ${ }^{23}$ Cantonal Hospital Baselland, University of Basel, Basel, Switzerland.
} 


\section{ONLINE METHODS}

Study populations and ethics information. The 4,484 patient plasma samples (4,515 including those later excluded for non-HIV-1-specific inhibitory activity in plasma; 3,078 male, 1,406 female; see Supplementary Table 1) analyzed in the current study were derived from samples stored in the biobanks of the Swiss HIV Cohort study (SHCS) and the Zurich Primary HIV Infection Study (ZPHI). The SHCS is a prospective, nationwide, longitudinal, noninterventional, observational, clinic-based cohort with semiannual visits and blood collections, enrolling all HIV-infected adults living in Switzerland ${ }^{29}$. The SHCS, founded in 1988, is highly representative of the HIV epidemiology in Switzerland, as it includes an estimated 53\% of all HIV cases diagnosed in Switzerland since the onset of the epidemic, $72 \%$ of all patients receiving ART in Switzerland, and 69\% of the nationwide registered AIDS cases ${ }^{29,43}$. The SHCS data are collected by the five Swiss university hospitals, two cantonal hospitals, 15 affiliated hospitals and 36 private physicians (listed in http://www.shcs.ch/180-health-care-providers). The SHCS is registered under the Swiss National Science longitudinal platform (http://www.snf.ch/en/funding/ programmes/longitudinal-studies/Pages/default.aspx\#Currently\%20supporte $\mathrm{d} \% 20$ longitudinal\%20studies). Detailed information on the study is available at http://www.shcs.ch.

The ZPHI is an ongoing, observational, nonrandomized, single center cohort founded in 2002 that specifically enrolls patients with documented acute or recent primary HIV-1 infection (ClinicalTrials.gov identifier NCT00537966) ${ }^{30}$.

The SHCS and the ZPHI were approved by the ethics committees of the participating institutions (Kantonale Ethikkommission Bern, Ethikkommission des Kantons St. Gallen, Comite Departemental d'Ethique des Specialites Medicales et de Medicine Communataire et de Premier Recours, Kantonale Ethikkommission Zürich, Repubblica et Cantone Ticino-Comitato Ethico Cantonale, Commission Cantonale d'Étique de la Recherche sur l'Être Humain, Ethikkommission beider Basel for the SHCS and Kantonale Ethikkommission Zürich for the ZPHI), and written informed consent was obtained from all participants. Further details on cohorts are provided in the Supplementary Note.

Patient data. Both SHCS and ZPHI maintain comprehensive, longitudinal, anonymous data collections for all participants, including extensive clinical and demographic data. The following parameters were used in the current study: longitudinal viral load and $\mathrm{CD} 4^{+}$cell measurements, clinical history (antiretroviral drug history, infection length, patient demographics) and nucleotide sequence data from genotypic antiretroviral drug resistance tests (GRT). See the Supplementary Note for details on how these parameters were recorded.

Time of HIV acquisition. The time of HIV acquisition was determined on the basis of the available data.

ZPHI. The time of HIV acquisition was determined for each individual enrolled in the ZPHI through consideration of clinical and laboratory data such as known risk situations, appearance of first symptoms, earlier negative test results, avidity assays and western blot results (negative, indeterminate and seroconversion date) as described elsewhere ${ }^{44}$.

SHCS. For the SHCS we used a hierarchical approach to estimate the infection date on the basis of indicators of varying reliability. The following methods were used with decreasing priority to yield the maximal accuracy for infection dates possible with the available data:

1. Defined primary infection: for 1,008 of the 4,484 patients, either seroconversion dates (negative and positive HIV screening test less than 1 year apart) or a diagnosis of a primary infection were available. We used the midpoint between the dates of the negative and positive tests or, if known, the date of the primary infection as the estimated infection date for these individuals.

2. Defined recent infection: an additional 544 patients had a treatmentnaive genotypic resistance test within the first year after diagnosis with low diversity (less than $0.5 \%$ of ambiguous nucleotides) and $\mathrm{CD} 4^{+}$cell counts above 200 at registration; on the basis of existing data in the literature ${ }^{34,45,46}$, we interpreted these as recent infections and used the diagnosis date as an estimate for the infection date.

3. For an additional 2,803 patients, we used infection-date estimates based on a back-calculation method ${ }^{47}$ using $\mathrm{CD} 4^{+}$cell counts and their slopes when available.
4. For the remaining 129 patients, no accurate dating was available. For these individuals the date of diagnosis was used as substitute for the infection date, which allowed us to define at least a minimal infection length. These $129 \mathrm{pa}-$ tients were assigned to group $1(N=37)$, group $3(N=38)$ or group $5(N=54)$. As the estimated infection date for these 129 individuals is a minimum estimate, the classification of individuals assigned to group 5 is accurate. Knowledge of an earlier infection date would not change their classification, group 5 already has the longest infection duration. We nevertheless verified that data from these 129 individuals did not influence our observations. The $P$ values reported in Supplementary Table 6 changed only marginally when the 129 individuals were excluded from the analysis. No additional significant observations were altered by inclusion or exclusion of this group (data not shown). We therefore opted to include these individuals in the final analysis. To exclude confounding influences from differential precision in the time of HIV acquisition on our data analysis, we verified that parameters identified in the complete cohort (Figs. 3 and $\mathbf{4}$ ) were detected in individuals with defined primary or recent infection $(N=1,552$, corresponding to categories 1 and 2 above; data on all parameters were available for testing for 1,476 ) and those for whom the infection time point was less precise and had to be estimated by the various strategies listed above $(N=2,932$, corresponding to categories 3 and 4 above; for 2,603 of whom all parameters were available for testing) (Supplementary Table 10).

Defining duration of HIV-1 exposure. The duration of HIV-1 exposure (net virus replication) was defined for each individual by accounting for all periods with untreated HIV-1 infection from the estimated time of HIV acquisition onward. For this, treatment regimens and treatment interruptions were evaluated for all included individuals, and a cumulative time off ART was calculated for each patient. Time periods covering the observation periods within the cohorts reflect exact accounts of time. Time periods before entry into the studies are approximate values based on the estimated dates of HIV acquisition (see above).

In a sensitivity analysis, we restricted the population to patients with at least 3 years of untreated infection (Supplementary Table 11). On the basis of our finding that infection time has no significant effect after 3 years of infection (Fig. 3), this restriction indicates the impact of other parameters while largely excluding the impact and potential confounding of infection time. To be conservative, this restriction was based on infection time calculated with the time of diagnosis instead of the estimated date of infection as a starting point. This thereby ensures that all included patients have experienced at least 3 years of untreated infection (independently of the estimation method used for the date of infection).

Patient selection, samples size and stratification. Individuals included in our screen had to meet several predefined criteria that were installed for the purpose of the current analysis and planned follow-up studies. We made no sample size restriction; all available samples that fulfilled the criteria were included.

1. Time of HIV-1 acquisition had to be known or predictable to define the length of untreated HIV-1 infection (see Supplementary Note).

2. The patients had to have experienced a minimum of 1 year of untreated HIV-1 infection.

3. Plasma samples off ART (for the neutralization studies conducted in the current study) and PBMCs (to allow isolation of bnAbs in follow-up studies) had to be available. If the sample was taken during a treatment interruption, we required that the sampling time was at least $90 \mathrm{~d}$ after the start of the interruption (to avoid residual effects of ART).

4. Because a main interest of our study was to define parameters of bnAb induction that may also be relevant during vaccination and thus in healthy immune systems, we restricted our analysis to patients at disease stages before AIDS, selecting only plasma samples before a stage $C$ event, as defined by the 1993 revised classification of the Centers for Disease Control and Prevention $^{48}$, and from time points with CD4 levels above 200 cells $/ \mu \mathrm{l}$.

5. The selected plasma sample had to have a detectable viral load. Although this potentially excludes elite controllers, the measure limits chances of residual ART being present and will allow virus envelope cloning from the same time point at which bnAb activity was identified in follow-up studies. 
6. Plasma had to be free of unspecific inhibitory activity as determined by inhibition of MuLV envelope pseudotyped virus.

Stratification. Broad neutralizing responses have been shown to preferentially develop after extended periods of virus replication and may even decline at later time points ${ }^{23}$. To obtain a global picture of bnAb frequency in HIV infection and address the timing aspects, we selected individuals and assigned them into one of three groups according to the length of HIV-1 exposure. Each individual was represented only once.

Group 1 ( $\geq 1$ year $<3$ years HIV-1 exposure). Individuals who were documented with untreated HIV infection (detectable viral load, off ART) for a minimum of 1 and a maximum of 3 years of infection. If multiple samples from one patient were available and fell into group 1, the sample taken closest to the 2-year time point was chosen.

Group 3 ( $\geq 3$ years $<5$ years HIV-1 exposure). If multiple samples from one patient were available and fell into group 3 , the sample taken closest to the 4-year time point was chosen.

Group 5 ( $\geq 5$ years $H I V-1$ exposure). If multiple samples from one patient were available and fell into group 5 , the sample taken closest to the 5 -year time point was chosen.

Blinding. Personnel who conducted the neutralization screen had no information on patient demographics at the time of analysis.

Reagents. Properties of monoclonal antibodies used in this study are listed in Supplementary Table 3.

Cells. 293T cells were obtained from the American Type Culture Collection (ATCC) and TZM-bl reporter cells ${ }^{49}$ through the NIH ARP. Both cell lines were cultured in DMEM containing 10\% FCS and antibiotics and regularly checked for the absence of mycoplasma.

Virus screening panels. Properties and sources of plasmids encoding the envelope genes of all HIV-1 strains included in the screening panels are listed in Supplementary Table 2 . We selected viruses of the primary 8 -virus screening and secondary 40-virus screening virus panels (Supplementary Table 2) on the basis of published bnAb neutralization data (CATNAP) $)^{50}$ and additional bnAb neutralization studies (Supplementary Table 4). In both virus panels we sought to include strains from different subtypes that allow both a sensitive detection of bnAb activity and a basic dissection of main bnAb categories based on neutralization fingerprint analysis. For the production of Env pseudovirus stocks, $293 \mathrm{~T}$ cells were transfected with the luciferase reporter HIV-1 pseudotype vector pNLlucAM and env expression plasmids as described ${ }^{51}$.

V2 envelope point mutations used to probe V2-glycan bnAb specificity (C1080.c3 N160D, CNE5 N160D, WITO K168E; Supplementary Fig. 6) were generated by site-directed mutagenesis (Agilent QuikChange II XL) according to the manufacturer's instructions. All point mutated envelopes were sequenced in house by Sanger sequencing to confirm presence of the desired mutations and absence of unintended sequence changes.

Primary neutralization screen. In the primary 8 -virus neutralization screen, neutralization capacity of patient plasma against a panel of eight divergent HIV-1 strains was assessed in a 384-well Env pseudovirus luciferase reporter assay on TZM-bl cells. Briefly, a 1/150 dilution of heat-inactivated plasma was preincubated with the respective virus for $1 \mathrm{~h}$ before infection of target cells. Neutralization activity was calculated as the reduction in infectivity (measured as relative light units luminescence evoked by the luciferase reporter) compared to infection in absence of plasma. Samples with confirmed activity against MuLV Env pseudovirus above 20\% were excluded.

Scoring neutralization activity using the primary screen. To evaluate breadth and potency, we used a scoring of the neutralization activity measured in the 8 -virus screen. Neutralization activity of plasma against a specific virus received a score of 0 when neutralization activity was below $20 \%$, a score of 1 when neutralization activity ranged between $20 \%$ and $<50 \%$, a score of 2 for neutralization between $50 \%$ and $<80 \%$ and a score of 3 for neutralization $\geq 80 \%$. Plasmas were then ranked by the sum of scores against all eight viruses to reflect their potency and breadth $(\mathbf{F i g} . \mathbf{1 b}, \mathbf{c})$. The maximum cumulative neutralization score a plasma could reach was 24 .

40-virus multiclade neutralization screen. The top-scoring bnAb containing plasmas (scores $\geq 13, N=105$; Supplementary Table 5) in the primary 8 -virus screen were subjected to a more in-depth characterization of neutralization potency and breadth using a panel of 40 genetically diverse Env pseudoviruses representing important subtypes and circulating recombinant forms (Supplementary Table 2). Serial dilutions of plasma samples starting at dilution 1/100 were assessed for neutralization activity and the reciprocal, half-maximum neutralization titer $\left(\mathrm{NT}_{50}\right)$ for each plasma virus combination defined. On the basis of these neutralization data, a final ranking of the top $105 \mathrm{bnAb}$ plasma samples was performed according to the geometric mean $\mathrm{NT}_{50}$ value of each plasma across all 40 viruses. For the calculation of this overall geometric mean, a value of 100 (the lowest dilution tested) was used for all plasma samples that did not reach a $\mathrm{NT}_{50}$ at this concentration. To reflect potency of the plasmas we additionally determined the geometric mean $\mathrm{NT}_{50}$ across all neutralized strains (Fig. 2 and Supplementary Table 5).

Definition of cross-, broad and elite neutralization activity. In recent years a consensus in the field has been reached that approximately $10-25 \%$ of individuals develop neutralization breadth ${ }^{17}$ and an estimated $1 \%$ of individuals generate highly potent bnAb activity, termed elite neutralization activity ${ }^{10,12,13,17,18,41,52-57}$. Yet, thus far no standardized classification methods have been installed to allow a unified definition of what precisely constitutes a broad or elite response. Moreover, as virus panels used for definition of breadth differ in size and composition, direct comparisons between studies remain difficult.

For this study, we needed a simple ranking system based on the 8-virus screen that allowed us to compare neutralization activity with low, medium and high breadth and potency to define parameters that influence development of high and low neutralization breadth. To this end we ranked the neutralization activities by setting threshold scores that best reflect the frequencies of expected elite and broad activity described in the literature ${ }^{10,12,13,17,18,41,52-57}$ and classified plasma with scores of 15 and higher as elite neutralization, scores of 10-14 as broad neutralization, scores of 5-9 as cross-neutralization and scores below 5 as no or weak neutralization activity. These thresholds provided a simple grading system: to be ranked as a cross-neutralizer, a plasma had to exhibit activity against at least two of the eight panel viruses (for example, one virus neutralized with $>80 \%$ and one with $>50 \%$; or more viruses with weaker potency); to be classified as broad it had to exhibit activity against at least four of the panel viruses (for example, three viruses with $>80 \%$ and one with $>20 \%$ ); to be classified as elite it had to exhibit potent activity against at least five of the panel viruses (for example, five viruses with $>80 \%$ ). This scoring system proved valid in identifying high neutralization breadth, as highlighted by activity of the top neutralizing plasmas (scores $\geq 13 ; N=105$ ) in the 40 -virus multiclade panel.

Prediction of bnAb epitope specificity in plasma. Neutralization patterns of bnAbs with defined epitopes against diverse virus panels can be used to establish neutralization fingerprints and delineate the epitope specificities in polyclonal plasma responses ${ }^{39,40}$. To derive information on the type of bnAb specificity evoked by the top $105 \mathrm{bnAb}$ inducers, we used the 40-virus multiclade panel to establish neutralization fingerprints of bnAbs with known epitope specificities and the top 105 plasmas (Supplementary Tables 4 and 5). Only bnAbs that neutralized ( IC $_{50}<50 \mu \mathrm{g} / \mathrm{ml}$ ) at least $50 \%$ of the panel viruses were included (Supplementary Table 4), and these were grouped by the HIV-1 Env epitope they target: V2 glycans (PG9, PG16, PGDM1400, PGT145), V3 glycans (PGT121, PGT128, PGT130), CD4bs (VRC01, PGV04), interface (PGT151) and MPER (2F5, 4E10, 10E8). From these data, delineation of plasma antibody specificities can be achieved by a variety of strategies. We chose a comparison of four different methods for the prediction of bnAb specificities and included a sensitivity analysis to verify the predictions (Fig. 5 and Supplementary Figs. 3-5).

To delineate the bnAb specificity of plasma antibodies in the maximalSpearman-based prediction (MSBP) method (Fig. 5 and Supplementary Figs. 3-5), a plasma was assigned to the bnAb with the most similar neutralization fingerprint, where similarity was measured using the Spearman correlation 
of the $\mathrm{IC}_{50}$ values of the bnAbs and the inverse $\mathrm{NT}_{50}$ values of the plasmas. If the fingerprint similarity with none of the included bnAbs was greater than 0.4 (a common threshold for moderate-strong correlation $)^{58}$ then no prediction was made.

To probe the validity of the predictions made with MSBP, we used three alternative methods (Supplementary Figs. 4 and 5).

1. The maximal-Pearson-based prediction (MPBP) follows the principle of MSBP with the exception that the Pearson correlation of the $\log \left(\mathrm{IC}_{50}\right)$ values of the bnAbs and the inverse $\log \left(\mathrm{NT}_{50}\right)$ values of the plasmas were used instead of the Spearman correlation coefficient.

2. A recent method developed by Georgiev et al. ${ }^{40}$ utilizes the rank-order of neutralization potencies of plasmas and reference antibodies to delineate plasma antibody specificities. We employed this analysis essentially as described ${ }^{40}$ and termed it delineation analysis Georgiev (DAG) in our comparative analysis. As our panel contained only a few bnAbs per class, we considered a version of the DAG algorithm where each bnAb was allowed to form its own cluster.

3. For a hierarchical clustering-based prediction (HCBP), we used a Spearman correlation matrix of all neutralization values (Supplementary Fig. 3) for plasmas and bnAbs as an input from which a UPGMA (unweighted pair group method with arithmetic mean) tree was derived. The UPGMA tree was computed on the basis of the Euclidean distance measure applied to the correlation matrix with the restriction of keeping plasmas and bnAbs separate. The subtree for plasmas was then cut into a predefined number (7) of clusters. These clusters correspond to groups of plasmas with similar correlation patterns to other plasmas and known bnAbs. As HCBP per se does not link a cluster to a specific bnAb type, we refer for interpretation purposes to that cluster with the largest overlap with MSBP-predicted CD4bs plasmas (overlap measured by adjusted Rand index) as CD4bs clusters and accordingly for the remaining bnAb specificities.

To assess the robustness of the defined bnAb type clusters in the four methods we performed two separate analyses (Supplementary Figs. 4a and 5a). In the first (Supplementary Fig. 4a), MSBP was set as reference, and thus we restricted the comparison to those 82 plasma samples for which MSBP could make a prediction. In the second robustness analysis (Supplementary Fig. 5a), MSBP was performed without a cutoff so that all 105 plasma samples were linked with the bnAb type for which they had the highest association, irrespective of the strength of correlation. Although this setting also assigns a bnAb specificity for very weak correlations and should thus not be used for a final specificity prediction, it enables a sensitivity analysis across all 105 plasma samples with all four prediction methods and to assess the impact of the cutoff in the MSBP method. For both settings, cluster robustness was evaluated using the adjusted and unadjusted Rand index (Supplementary Figs. $\mathbf{4 b}, \mathbf{c}$ and $\mathbf{5 b}, \mathbf{c}$ ). Both analyses (with and without restriction in MSBP) showed that V2-glycan- and CD4bs-specific antibodies were robustly predicted, whereas predictions for V3- and MPER-specific antibodies varied to a greater extent between the methods.

In a further sensitivity analysis, we probed the agreement of the four prediction methods by investigating the influence of the infecting subtype on the type of bnAb (Supplementary Figs. 4d and 5d). Using Fisher's exact test, we found that for both analyses, with and without restriction in MSBP, all four prediction methods showed a similar impact of viral subtype on bnAb type, thus highlighting that the findings do not depend on the prediction method.

Confirmation of predicted V2-glycan cluster plasma using V2 mutant viruses. Neutralization of bnAbs and the top 105 plasma samples was assessed against three pairs of wild-type and V2 mutant viruses (C1080c3/C1080.c3 N160D, CNE5/CNE5 N160D and WITO/WITO K168E) that are known to affect efficacy of V2-glycan bnAbs Pg9, Pg16 or PGT145 (Supplementary Fig. 6). Neutralization assays with Env pseudoviruses were performed using the TZM-bl assay as described above, and Wilcoxon signed-rank tests were used to compare the differences between the $\log _{10}\left(\mathrm{NT}_{50}\right)$ values of wild-type and mutant virus for each bnAb specificity.
Statistical analysis. Statistical analyses were performed in STATA (version 14) and in $\mathrm{R}$ (version 3.1.1). Odds ratios were computed using univariable and multivariable multinomial logistic regression with breadth of neutralization response as the outcome variable (categories: no neutralization (baseline), cross-neutralization, broad neutralization, and elite neutralization) in Figures $\mathbf{3}$ and $\mathbf{4}$ and Supplementary Table $\mathbf{6}$. In these analyses, viral load and CD4 level at time of sampling were included as continuous variables; duration of untreated infection, HIV-1 subtype, mode of transmission, ethnicity, and sex as categorical variables. These seven parameters were included and controlled for in all multivariate analyses listed in Figures $\mathbf{3}$ and $\mathbf{4}$ and in Supplementary Table 6. In Figure 3f,g and Supplementary Table 6, diversity was included as an additional parameter. The regression analyses performed and referred to in the text and figures are summarized in Supplementary Figure 2 and Supplementary Tables 6 and 14.

Supplementary Table 6 also contains additional regression analyses performed to control for potential influences of diversity on the findings. In the analysis for the effect of diversity the fraction of ambiguous nucleotides was added as a continuous covariable (see above).

To verify findings obtained with multinomial logistic regression and to perform analyses on smaller subgroups of our cohort that required methods with higher statistical power, we performed multivariable linear regression with the neutralization score as a continuous outcome variable. Supplementary Table 7 shows an analysis with linear regression; Supplementary Tables 7-11 show analyses with Tobit linear regression. The latter provides a better fit for this type of data, as the distribution of scores is strongly truncated. In particular, we chose for the Tobit regression a lower limit (i.e., right truncation) of 0 to take into account the fact that scores below 0 are not possible (score 0 is the most frequently observed score value). Using Tobit regression we further assessed the influence of specific CD4 levels (Supplementary Table 8) and individual env subtypes (Supplementary Table 9) and ruled out confounding influences of the assessment method for the time of HIV acquisition in our cohort on the reported results (Supplementary Table 10).

We used the Rand index to compare the overlap of the antibody prediction according to the different prediction methods. For each of the bnAb types (V2 glycan, V3 glycan, CD4bs, MPER) and each prediction method (MSBP, MPBP, DAG, HCBP), we classified the plasmas into two groups-those belonging to the given bnAb type according to a given method and those not-and determined the overlap between this grouping across the different methods using the unadjusted and the adjusted Rand index ${ }^{59}$ (Supplementary Figs. $4 b, c$ and $5 b, c)$.

Owing to the relatively small number of specificity predictions, the association of bnAb epitope specificity and subtype was tested with Fisher's exact test (Fig. 5 and Supplementary Figs. $4 \mathbf{d}$ and $\mathbf{5 d}$ ) and univariable and multivariable exact logistic regression, where we considered only ethnicity and subtype in the multivariable model (Fig. 5 and Supplementary Table 9).

Source data. Source Data for Figure 1 were also used for Supplementary Figure 1; data for Supplementary Figures 3-6 are listed in Supplementary Data Sets 1-4, respectively.

43. Yang, W.L. et al. Assessing the paradox between transmitted and acquired HIV type 1 drug resistance mutations in the Swiss HIV Cohort Study from 1998 to 2012. J. Infect. Dis. 212, 28-38 (2015).

44. Rieder, P. et al. HIV-1 transmission after cessation of early antiretroviral therapy among men having sex with men. AIDS 24, 1177-1183 (2010).

45. Ragonnet-Cronin, M. et al. Genetic diversity as a marker for timing infection in HIV-infected patients: evaluation of a 6-month window and comparison with BED. J. Infect. Dis. 206, 756-764 (2012).

46. Andersson, E. et al. Evaluation of sequence ambiguities of the HIV-1 pol gene as a method to identify recent HIV-1 infection in transmitted drug resistance surveys. Infect. Genet. Evol. 18, 125-131 (2013). 
47. Taffé, P. \& May, M. A joint back-calculation model for the imputation of the date of HIV infection in a prevalent cohort. Stat. Med. 27, 4835-4853 (2008).

48. Castro, K.G. et al. 1993 Revised classification system for HIV infection and expanded surveillance case definition for AIDS among adolescents and adults. MMWR 41, RR-17 (CDC, 1992).

49. Wei, X. et al. Antibody neutralization and escape by HIV-1. Nature 422, 307-312 (2003).

50. Yoon, H. et al. CATNAP: a tool to compile, analyze and tally neutralizing antibody panels. Nucleic Acids Res. 43, W213-W219 (2015).

51. Rusert, P. et al. Interaction of the gp120 V1V2 loop with a neighboring gp120 unit shields the HIV envelope trimer against cross-neutralizing antibodies. J. Exp. Med. 208, 1419-1433 (2011).

52. Walker, L.M. et al. A limited number of antibody specificities mediate broad and potent serum neutralization in selected HIV-1-infected individuals. PLOS Pathog. 6, e1001028 (2010).
53. Mikell, I. et al. Characteristics of the earliest cross-neutralizing antibody response to HIV-1. PLoS Pathog. 7, e1001251 (2011).

54. Binley, J.M. et al. Profiling the specificity of neutralizing antibodies in a large panel of plasmas from patients chronically infected with human immunodeficiency virus type 1 subtypes B and C. J. Virol. 82, 11651-11668 (2008).

55. Dhillon, A.K. et al. Dissecting the neutralizing antibody specificities of broadly neutralizing sera from human immunodeficiency virus type 1-infected donors. J. Virol. 81, 6548-6562 (2007).

56. Hraber, P. et al. Impact of clade, geography and age of the epidemic on HIV-1 neutralization by antibodies. J. Virol. 88, 12623-12643 (2014).

57. Hraber, P. et al. Prevalence of broadly neutralizing antibody responses during chronic HIV-1 infection. AIDS 28, 163-169 (2014).

58. Campbell, M.J. \& Swinscow, T.D.V. Statistics at Square One (Wiley-Blackwell/BMJ Books, 2009).

59. Rand, W.M. Objective criteria for evaluation of clustering methods. J. Am. Stat Assoc. 66, 846-850 (1971) 